\title{
Optimising the Design of a Broadband Josephson Junction TWPA for Axion Dark Matter Search Experiments
}

\author{
Javier M. Navarro ${ }^{\mathrm{a}}$ and Boon-Kok Tan ${ }^{\mathrm{a}}$ \\ a Dept. of Physics (Astrophysics), Univ. of Oxford, DWB Keble Road, OX1 3RH, Oxford, U.K.
}

\begin{abstract}
Broadband ultra-low noise amplification is important for many fundamental physics experiments. In our case, we aim to develop a quantum limited Josephson Travelling Wave Parametric Amplifier (JTWPA) for dark matter search experiments. In this paper, we focus on the development of the JTWPA, in particular to optimise the performance of the amplifier with a simplified fabrication prospect. We present our methodology for the optimisation process, focusing on three important aspects, namely utilising the minimal number of tunnel junction required, maximising the operational bandwidth and achieving a $50 \Omega$ characteristic impedance. We first explore the relations between the important circuit parameters of the JTWPA and its performance indicators. Using the information obtained, we perform our optimisation process to search for the optimal design parameters. We then compare the gain bandwidth performance of the different optimised models, and present our findings with further analyses. We demonstrate that our optimised model requires $4 \times$ less tunnel junctions compared to the conventional model, and we are able to improve the operational bandwidth by $68 \%$ while maintaining the characteristic impedance of the device at $50 \Omega$.
\end{abstract}

Keywords: Parametric Amplifier, Travelling Waves, Josephson Junctions, Axion Particle Dark Matter Search, Quantum Limited, Optimisation

\section{INTRODUCTION}

It is crucial to operate ultra-sensitive detection schemes at the fundamental quantum limit for axion dark matter particle search experiments. In the $10^{-6}$ to $10^{-3}$ electron-volt mass range, these experiments utilise high-Q tunable cavity inside a strong magnetic field environment to probe the relic axions at different microwave frequencies. Present state-of-the-art axion search experiments such as ADMX (the Axion Dark Matter eXperiment) $)^{1,2}$ and HAYSTAC (the Haloscope At Yale Sensitive To Axion CDM) ${ }^{3}$ utilise Josephson parametric amplifiers (JPAs) to amplify the signal with close to, and beyond, the standard quantum limit of added noise. ${ }^{4}$ Although this approach is highly feasible, the sensitivity deteriorate with higher frequency as the quantum noise of the amplifier scales with frequency. Furthermore, each of these cavity/JPA receivers can only operate within very narrow bandwidth, hence many receivers have to be assembled adjacent to each other to cover the large axion mass range. Many efforts had been made and proposed to accelerate the scanning rate while retaining the quantum limited sensitivity of the receiver. One of the leading proposal is by using an array of resonance qubits as single microwave photon detectors, ${ }^{5-8}$ and readout the array with a broadband travelling wave parametric amplifier (TWPA) such as Josephson (J-) TWPA ${ }^{9}$ or kinetic inductance (KI-) TWPA. ${ }^{10}$ These newly developed TWPA can reach the quantum noise limit with high gain over much larger bandwidth ( $\mathrm{GHz}$ instead of $\mathrm{MHz})$, hence dramatically improve the frequency coverage for axion search.

It is well established that the state of a qubit is inherently very sensitive to external disturbance. ${ }^{11} \mathrm{Tt}$ can therefore be engineered to detect the weak axion-induced photon either by measuring the quantum coherence or the phase/state of the qubit using a JPA, since in axion search one is only interested in detecting the presence of the generated photons, not their phase. However, it is not conceivable to cover a reasonable fraction of possible axion masses using such narrowband amplifier. By replacing a series of these narrow band qubit-JPAs receivers with a single broadband TWPA to readout an array of qubits tuned at slightly different resonance frequencies, as illustrated in Fig. 1, we shall be able to accelerate the search without sacrificing the sensitivity limit. This

Further author information: (Send correspondence to B-. K. Tan)

B-. K. Tan: E-mail: boonkok.tan@physics.ox.ac.uk, Telephone: +44 1865273303 
(a)

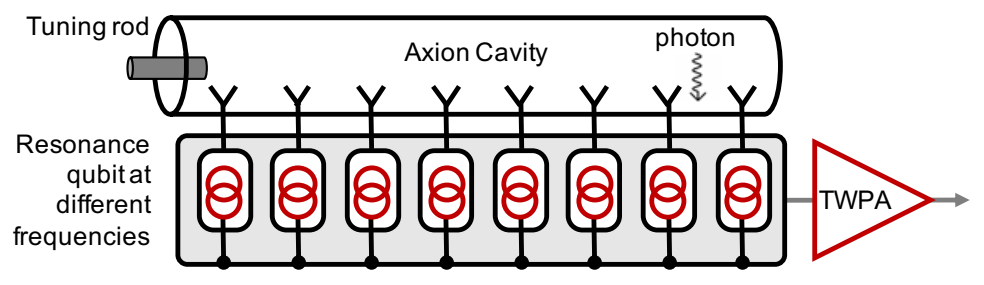

(b)

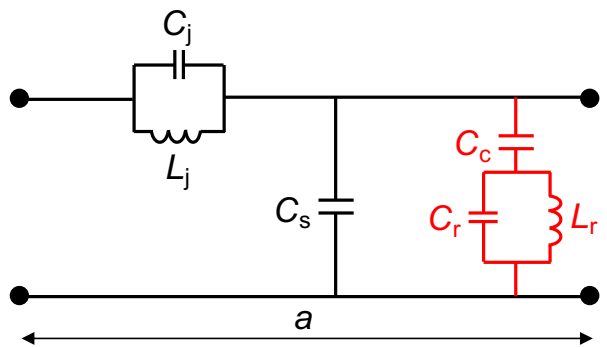

Figure 1. (a) Diagram illustrating the proposed single microwave photon detection scheme utilising an array of qubit installed inside a tunable cavity and readout using a broadband TWPA. (b) Circuit diagram of the unit cell of our JTWPA The $L C$ resonator used to achieve exponential gain is shown in red colour.

approach also allow us to simplify the receiver backend architecture with a single readout electronic chain, further reducing the size and complexity of the experiment.

Apart from the broadband nature of this detection scheme, one other important advantage is that the same architecture can be deployed for much higher frequencies operation, potentially into the millimetre wave regime. Furthermore, as the qubit and the TWPA can be fabricated using the same photolithography technique, it could be possible to further integrate the two within a single platform, and thereby better engineer the interaction between the qubit detector and the readout amplifier, hence improving the performance of the complete receiver system.

In this paper, we focus on the development of a Josephson TWPA to work in tandem with the superconducting qubit array. In particular, we aim to improve the performance of the JTWPA while kept the fabrication process as simple as possible to achieve high yield. We search for the optimal set of design parameters that can achieve three important goals: firstly, the minimum number of tunnel junctions required to achieve a certain peak gain; secondly maximise the operational bandwidth of the JTWPA and; finally achieving a characteristic impedance $Z_{0}=50 \Omega$ to match with the wider circuitry and minimise the gain variation effect. In the following, we shall discuss in detail our optimisation process, and compare the predicted performance of several different optimised models with further analyses and discussions.

\section{DESIGN METHODOLOGY}

For this project, we aim to design a JTWPA that has at least $20 \mathrm{~dB}$ gain from $8-12 \mathrm{GHz}$ with a compression point around $-100 \mathrm{dBm}$. The main transmission line of our JTWPA will be formed using superconducting aluminium (Al) embedded with a series of $\mathrm{Al}-\mathrm{AlO}_{\mathrm{x}}$ (aluminium oxide) tunnel junctions, fabricated on a sapphire substrate. We expect that the JTWPA will be operating at a base temperature of $10 \mathrm{mK}$.

In order to simplify the design and optimisation process, the unit cell of our JTWPA is represented with a two-port circuit (lossless transmission line shunted with a capacitor) coupled with a tunnel junction modelled as a simple LC circuit in parallel, as shown in Fig $1(\mathrm{~b})$. We neglect the geometrical inductance $L_{\text {geo }}$ of the transmission line, since for low compression point operation, the required junction inductance $L_{\mathrm{j}}$ is often much larger i.e., $\left(L_{\mathrm{j}} \gg L_{\mathrm{geo}}\right)$. At later stages, we coupled these junction cells with a superconducting $L C$ resonator for dispersion engineering to achieve higher gain. These unit cells were cascaded to form the circuit model of the entire amplifier chip, with the gain-bandwidth product of the JTWPA calculated using the standard coupled mode equations $(\mathrm{CME})^{12,13}$ in the four-wave mixing (4WM) regime.

\section{OPTIMISATION OF JTWPA WITHOUT DISPERSION ENGINEERING}

The circuit model of our JTWPA depicted in Fig 1 (b) is in fact identical to the model presented by O'Brien et. al. ${ }^{13}$ (hereafter the original model). We based our design on this model as it is the simplest theoretical model required to form a high gain JTWPA, and we aim to optimise this design and compare the final performance with the result presented by O'Brien et. al. Although it is well known that a functional TWPA requires a mechanism for dispersion control and harmonics suppression, we begin our design process by excluding the $L C$ resonators and use only the capacitively shunted tunnel junctions as our basic unit cells. 


\subsection{Minimising the Number of Tunnel Junctions Required}

The first aspect we investigated is the minimal number of tunnel junctions required to achieve $9.3 \mathrm{~dB}$ peak gain, a value similar to the original model, using the same pump current to critical current $\left(I_{\mathrm{p}} / I_{c}=0.039\right)$ and signal current to pump current $\left(I_{\mathrm{s}} / I_{\mathrm{p}}=0.5\right)$ ratios presented by O'Brien et. al. Minimising the total number of junctions required is important because a large number of junctions connected in series could potentially lower the yield, unnecessarily lengthen the transmission line which would induce higher loss and noise, and more importantly the JTWPA will be rendered unusable if only one of the tunnel junction is open-circuited.

Assuming a transmission line with $Z_{0}=50 \Omega$ and the requirement to achieve a compression point of $P_{1 \mathrm{dBm}}=$ $-100 \mathrm{dBm}$, we calculated the required $L_{\mathrm{j}}$ to be $101.7 \mathrm{pH}$, which translates to a critical current value of $3.24 \mu \mathrm{A}$. We further assume that the physical length of a unit cell $a=10 \mu \mathrm{m}$, following the value reported in White et. al. ${ }^{14}$ Once $L_{\mathrm{j}}$ and $a$ are fixed, we explore the effect of the remaining parameters in our unit cell circuit, namely the junction capacitance $C_{\mathrm{j}}$ (which related to the junction area) and the shunt capacitance $C_{\mathrm{s}}$.

First, we calculate the circuit cutoff frequency $\left(f_{\mathrm{c}}\right)$ using $\omega_{\mathrm{c}}=2 \omega_{0} / \sqrt{1+4 \omega_{0}^{2} / \omega_{\mathrm{j}}^{2}}$, where $\omega_{\mathrm{j}}=\sqrt{L_{\mathrm{j}} C_{\mathrm{j}}}$ is the plasma frequency of the Josephson junction (JJ) and $\omega_{0}=\sqrt{L_{\mathrm{j}} C_{\mathrm{s}}}$ the cutoff frequency of the transmission line respectively. In conventional JTWPAs reported to date, ${ }^{9,14,15}$ the higher harmonics suppression was achieved by relying on the $f_{\mathrm{c}}$ of the circuit being closer to the higher end of the operational bandwidth. This approach has a downside where the gain drops rapidly away from the central frequency, hence reducing the usable bandwidth (see Section 3.2). This is because the complex transmission parameter $S_{21}$ tents to diverge rapidly towards the cutoff frequency. Nevertheless, we opt for a similar approach since we aim to optimise the bandwidth performance in later section. Therefore, we set the $f_{\mathrm{c}}$ lower limit to around $3 \times$ the central frequency i.e., in our case $\sim 30 \mathrm{GHz}$, as smaller values would deteriorate the bandwidth performance without any further advantages. This lead us to a range of $C_{\mathrm{j}}$ that can be used, as shown in the white region in Fig. $2(\mathrm{a})$. Assuming $C_{\mathrm{s}}=150 \mathrm{fF}$, we can see that $C_{\mathrm{j}}$ should not exceed $240 \mathrm{fF}$ otherwise $f_{\mathrm{c}}<30 \mathrm{GHz}$. This set the upper limit for $C_{\mathrm{j}}$, while the lower limit of $70 \mathrm{fF}$ is limited by the feasibility of fabricating small junctions, which we expect needs to be larger than $1.0 \mu \mathrm{m}^{2}$. Note that the upper limit of $C_{\mathrm{j}}$ cited here (which corresponds to $\sim 3.5 \mu \mathrm{m}^{2}$ junction) is dependent on the value of shunt capacitance used, albeit not significantly deviate from the 200-250 $\mathrm{fF}$ range.

We next calculate the number of cells i.e., the number of Josephson junctions $\left(N_{\mathrm{JJ}}\right)$ required to achieve $9.3 \mathrm{~dB}$ peak gain. We explore a range of different shunt capacitance $C_{\mathrm{s}}$ values as before, within the previously fixed $C_{\mathrm{j}}$ range. The results are plotted in Fig. $2(\mathrm{~b})$. We observe that by increasing $C_{\mathrm{s}}$ we can effectively reduce $N_{\mathrm{JJ}}$
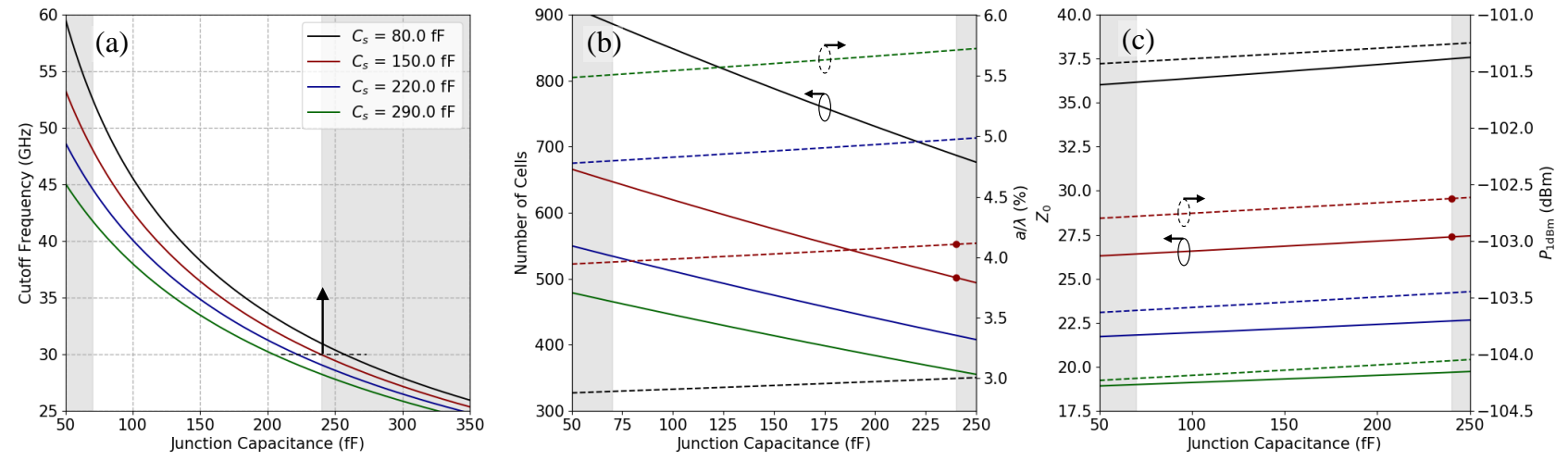

Figure 2. Impact of $C_{\mathrm{j}}$ and $C_{\mathrm{s}}$ with different circuit parameters for Model 1. Only four values of $C_{\mathrm{s}}$ are plotted for simplicity. The solid and dashed lines correspond to the left and right y-axis respectively. The colour coding used for different case of $C_{\mathrm{s}}$ is identical for all plots. (a) Influence of $C_{\mathrm{j}}$ and $C_{\mathrm{s}}$ on the circuit cutoff frequency, where the white region shows the range of feasible capacitance values. (b) Number of cells required to achieve $9.3 \mathrm{~dB}$ gain without dispersion engineering, and the $a / \lambda$ ratio in relation to $C_{\mathrm{j}}$ and $C_{\mathrm{s}}$. (c) Changes in characteristic impedance and compression point in relation to $C_{\mathrm{j}}$ and $C_{\mathrm{s}}$. 
e.g., using only $\sim 70 \%$ of the junctions if $C_{\mathrm{s}}$ is increased from 80 to $150 \mathrm{fF}$. This is due to the reduction in the phase velocity of the transmission line with increased $C_{\mathrm{s}}$, which in turn increases the interaction time between the signal and the pump waves, hence obtaining the same peak gain with a smaller number of junctions. We can therefore conclude that the $C_{\mathrm{s}}$ needs to be maximised to minimise $N_{\mathrm{JJ}}$.

JTWPAs are usually implemented using CPW technology, however the preference of having a high value of $C_{\mathrm{s}}$ suggests that other transmission line topologies such as inverted grounded CPW or microstrip might be more suitable, as higher $C_{\mathrm{s}}$ values can be achieved more easily. Microstrip line also have other advantages such as less radiative, easier to design etc, although the fabrication process will be slightly complicated with the need for multi-layer fabrication facilities. Nevertheless, even though CPW transmission line is simpler to fabricate with single layer fabrication, it potentially needs a large number of equipotential bridges across the CPW to ensure suppression of unwanted modes. ${ }^{16}$ Fabrication of these bridges would inevitably require multi-layer fabrication technique as well, and furthermore the existence of these bridges would also induce additional geometric shunt capacitance, hence provided another degree of freedom to achieve the required $C_{\mathrm{s}}$ value.

However, increasing $C_{\mathrm{s}}$ will have an impact on the electrical length $(\lambda)$ of the cell, represented here by the ratio of $a / \lambda$. The CME assumes that $a / \lambda \ll 1$ such that the JJs act as distributed elements in the transmission line. If this ratio is closer to 1 , this assumption is not valid anymore, and the junctions become discreet or lumped elements and the CME would fail to describe the wave propagation properly. Hence, this imposes an upper limit in $C_{\mathrm{s}}$, which we fixed to a ratio value of $a / \lambda \sim 4 \%$. From Fig. $2(\mathrm{~b})$, this essentially rules out the case for $C_{\mathrm{s}}=220 \& 290 \mathrm{fF}$. Since lower value of $a / \lambda$ simply increase the number of JJs within a certain physical transmission length without significant contribution to higher gain, this leads us to the optimal value of $C_{\mathrm{s}}=150 \mathrm{fF}$ where $a / \lambda$ is now close to the $4 \%$ range. Once $C_{\mathrm{s}}$ is determined, it is easier to find the optimal value of $C_{\mathrm{j}}$ by referring back to Fig. 2 (b). It is clear that higher $C_{\mathrm{j}}$ further reduce the required $N_{\mathrm{JJ}}$, while the changes in $a / \lambda$ is marginal. Increasing $C_{\mathrm{j}}$ from 70 to $240 \mathrm{fF}$ could further decrease $N_{\mathrm{JJ}}$ from 650 to 500 while $a / \lambda$ only increase slightly from 4.0 to $4.1 \%$.

This leads us to the set of parameters $\left(C_{\mathrm{s}}=150 \mathrm{fF}, C_{\mathrm{j}}=240 \mathrm{fF} \& f_{\mathrm{c}}=30 \mathrm{GHz}\right)$ for achieving the minimal $N_{\mathrm{JJ}}$ with a peak gain of $9.3 \mathrm{~dB}$, which we shall refer to as Model 1 for comparison in later sections. These parameters however result in a different $Z_{0} \neq 50 \Omega$ characteristic impedance and compression point, which in this case is now closer to $27 \Omega$ and $-102.6 \mathrm{dBm}$ as shown in Fig. 2 (c). Nevertheless, the compression point is not too far deviated from our intended value of $-100 \mathrm{dBm}$, but the decrease in $Z_{0}$ could potentially deteriorate the performance of the TWPA due to impedance mismatch with the wider $50 \Omega$ environment, which we will explore in later sections.

\subsection{Improving the Operational Bandwidth}

Instead of aiming to achieve minimal number of junctions, in this section we explore the limits of operational bandwidth with regards to the circuit parameters. This is because the number of junctions required in Model 1 is in fact $4 \times$ lower than most JTWPAs reported in literature, hence we investigate how the bandwidth performance can be improved if we relax the requirement on $N_{\mathrm{JJ}}$.

We begin our investigation with $L_{\mathrm{j}}$ set at $101.7 \mathrm{pH}$ similar to Section 3.1 and $C_{\mathrm{j}}$ at $70 \mathrm{fF}$, the lower limit of the range shown in Fig. 2. First, we plot the relation between $f_{c}$ and the operational bandwidth in Fig. 3 (a). We use the percentage bandwidth $(\% \mathrm{BW})$ as a unit of measurement here, where the edges of the band is defined as half of the peak gain in $\mathrm{dB}$ with a normalised frequency $\Delta f / f=\left(f_{\mathrm{s}}-f_{\mathrm{p}}\right) / f_{\mathrm{p}}$. As indicated earlier, indeed we observe that higher values of $f_{\mathrm{c}}$ will improve the bandwidth performance. Although the bandwidth is relatively insensitive to the value of $C_{\mathrm{s}}$, it is worthwhile noting that higher $C_{\mathrm{s}}$ also widen the bandwidth.

Starting with $C_{\mathrm{j}}=70 \mathrm{fF}$ and $C_{\mathrm{s}}=150 \mathrm{fF}$, from Fig. 2 (a) we see that this results in $f_{c} \sim 48 \mathrm{GHz}$, which corresponds to $50 \%$ bandwidth as indicated in Fig. 3 (a). We can further increase $f_{\mathrm{c}}$ to improve the bandwidth performance by reducing $L_{\mathrm{j}}$. Assuming we aim to double the bandwidth from $50 \%$ to $100 \%$, we need to increase $f_{\mathrm{c}}$ from 50 to $160 \mathrm{GHz}$. From Fig. 3 (b), this results in a junction inductance of $9 \mathrm{pH}$, approximately $10 \times$ smaller

\footnotetext{
*It is conventional to considered a structure feature smaller than 1/10 of a wavelength at the maximum usable frequency $\left(f_{\max }\right)$ as a discreet element. Assuming $f_{\max }=25 \mathrm{GHz}$ in our case to be on the safe side, this resulting in a value of $1 / 25$ of a wavelength at our central frequency, which corresponding to $a / \lambda=4 \%$.
} 

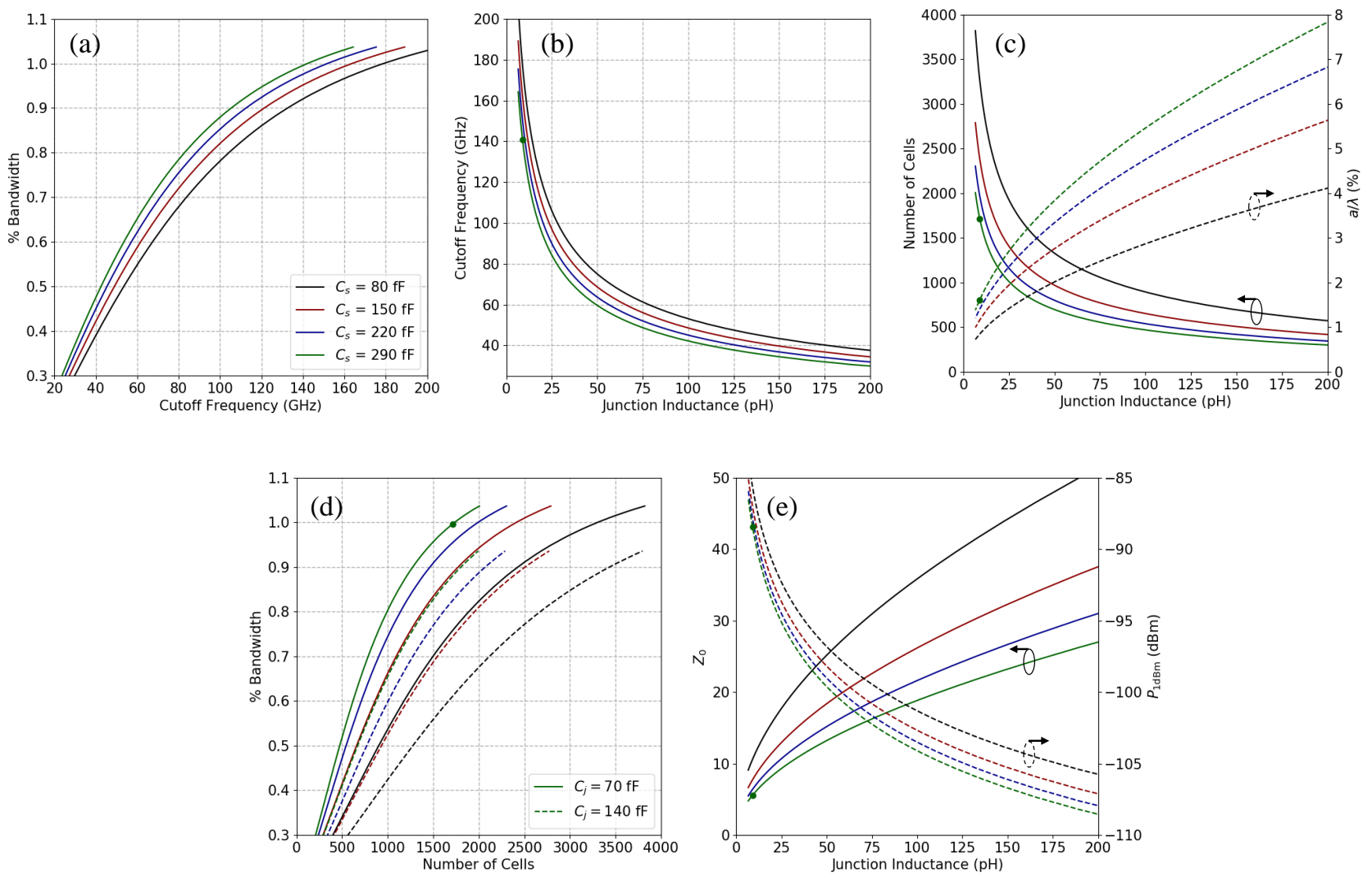

Figure 3. (a) The relation between $\% \mathrm{BW}$ and $f_{\mathrm{c}}$. Each point in this plot was produced by optimising $L_{\mathrm{j}}$ and $N_{\mathrm{JJ}}$ to achieve a peak gain of $9.3 \mathrm{~dB}$ with $C_{\mathrm{j}}$ fixed to $70 \mathrm{fF}$. (b) Plot showing how $L_{\mathrm{j}}$ influences $f_{\mathrm{c}}$ with fixed $C_{\mathrm{j}}$ value. (c) Plots of $N_{\mathrm{JJ}}$ and $a / \lambda$ against $L_{\mathrm{j}}$. (d) Relationship between $\%$ BW and $N_{\mathrm{JJ}}$. (e) Changes in characteristic impedance and compression point as a function of $L_{\mathrm{j}}$.

than the value used earlier. This inevitably increased the required number of junctions to 1,713, as shown in Fig. 3 (c), as lower $L_{\mathrm{j}}$ implies weaker non-linearity hence more junctions will be needed to achieve the same gain. Furthermore, lower $L_{\mathrm{j}}$ reduce the value of $a / \lambda$ substantially, which interestingly indicates that employing more JJs within a wavelength below $a / \lambda=4 \%$ point is not without benefit, as it helps to improve the bandwidth performance.

Therefore, there is a compromise between the choice of minimising $N_{\mathrm{JJ}}$ against broader bandwidth. This is best demonstrated in Fig. 3 (d) where the \%BW of the JTWPA increase approximately linearly with the required $N_{\mathrm{JJ}}$. Note that this plot is in fact a compressed way of illustrating the same information contained in the upper panels of Fig. 3 within a single plot, where $\% \mathrm{BW} \rightarrow f_{\mathrm{c}} \rightarrow L_{\mathrm{j}} \rightarrow N_{\mathrm{JJ}}$ (corresponding to Fig. 3 (a), (b) and (c) respectively). However, interestingly, it also illustrates the important of shunt capacitance $C_{\mathrm{s}}$ in this case., as the slope of the curve is steeper with higher $C_{\mathrm{s}}$. This indicates that the bandwidth improvement due increased $C_{\mathrm{s}}$ is more significant with smaller value of $N_{\mathrm{JJ}}$. This again shows the importance of high $C_{\mathrm{s}}$ value, a similar finding concluded in earlier section.

Although up until this point, we have not yet explore the effect of $C_{\mathrm{j}}$ in this scenario, but we can simply reproduce the same calculation for the $\% \mathrm{BW}-N_{\mathrm{JJ}}$ relation by doubling $C_{\mathrm{j}}$ to $140 \mathrm{fF}$ and observe the resulting changes. These are plotted as dashed lines in Fig. $3(\mathrm{~d})$, and it clearly shows that higher $C_{\mathrm{j}}$ deteriorate the bandwidth performance and require more junctions to achieve the same gain. Lower value of $C_{\mathrm{j}}$ is preferable here, but as we mentioned earlier, if $C_{\mathrm{j}}<70 \mathrm{fF}$, the junction size would need to be smaller than $1 \mu \mathrm{m}^{2}$, therefore this is in fact the optimal value of $C_{\mathrm{j}}$ in our case. 
To summarise, from Fig. 3 we show that we can achieve $100 \%$ bandwidth performance with the following set of parameters: $C_{\mathrm{s}}=290 \mathrm{fF}, C_{\mathrm{j}}=70 \mathrm{fF}, L_{\mathrm{j}}=9 \mathrm{pH}$ and $a / \lambda=1.6 \%$, which results in a cutoff frequency of $140 \mathrm{GHz}$ and $N_{\mathrm{JJ}}=1,713$. Hereafter, this optimised bandwidth model will be referred to as Model 2 . Finally, Fig. 3 (e) shows the resulting characteristic impedance and compression point of this model, where $Z_{0}$ is now extremely low at $5.6 \Omega$ and $P_{1 \mathrm{dBm}}$ is increased to $-88.5 \mathrm{dBm}$.

\subsection{Achieving $50 \Omega$ Characteristic Impedance Line}

As shown in the cases of Model 1 and 2, each optimisation process resulting in a lower characteristic impedance of $Z_{0} \neq 50 \Omega$, which is non-ideal for the JTWPA to operate in a commonly-used $50 \Omega$ system. To ensure that $Z_{0}$ remains at $50 \Omega$, we can further alter the value of either $C_{\mathrm{j}}, C_{\mathrm{s}}$ or $L_{\mathrm{j}}$.

Referring back to Fig. 2 (c), it is clear that $C_{\mathrm{j}}$ have little influence on $Z_{0}$, but the impact of $C_{\mathrm{s}}$ is significant. Unfortunately $C_{\mathrm{s}}$ is in inverse relation to $Z_{0}$, which is unfavourable as lower $C_{\mathrm{s}}$ would sacrifice the bandwidth performance and increase the required $N_{\mathrm{JJ}}$ as shown earlier in Section 3.1 and 3.2. Therefore, the only parameter left to influence $Z_{0}$ is $L_{\mathrm{j}}$, which related directly to the critical current value $I_{c}$. Since $Z_{0} \propto \sqrt{L_{\mathrm{j}}}$, we need to increase $L_{\mathrm{j}}$ i.e., decreasing $I_{c}$ to reach a higher value of $Z_{0}$. Assuming $C_{\mathrm{j}}$ fixed at $70 \mathrm{fF}$, we can infer from Fig. 3 (e) that the only case we can achieve $Z_{0}=50 \Omega$ within the plotted $L_{\mathrm{j}}$ range is if $C_{\mathrm{s}}=80 \mathrm{fF}$, corresponding to $L_{\mathrm{j}}=189.3 \mathrm{pH}$. Coincidentally, this results in the value of $a / \lambda=4 \%$ as shown in Fig. 3 (c), with the required number of junctions $N_{\mathrm{JJ}}=594$, and a $38 \%$ bandwidth.

This new design is hereafter referred to as Model 3 which effectively reduces the number of junctions while keeping $Z_{0}=50 \Omega$. It is obvious that increasing $L_{\mathrm{j}}$ would leads to smaller value of $N_{\mathrm{JJ}}$, but it comes with a price of reduced bandwidth. For example, if we refer to the $C_{\mathrm{s}}=220 \mathrm{fF}$ plots in Fig. $3(\mathrm{e})$, we would need $L_{\mathrm{j}}>300 \mathrm{pH}$ to reach $Z_{0}=50 \Omega$. This reduce $N_{\mathrm{JJ}}$ substantially, and since $N_{\mathrm{JJ}}$ is directly proportional to $\% \mathrm{BW}$, the bandwidth performance will therefore be limited. More importantly, from Fig. 3 (c), the ratio $a / \lambda$ is now way beyond $4 \%$, therefore we are risking the JJs be seen as lumped instead of distributed element by the propagating waves.

In order improve Model 3 with larger bandwidth, we could decrease both $L_{\mathrm{j}}$ and $C_{\mathrm{s}}$ further, as it is obvious that $C_{\mathrm{s}}<80 \mathrm{fF}$ will allow us to reach $Z_{0}=50 \Omega$ with lower $L_{\mathrm{j}}$, hence increase the cutoff frequency and thus the \%BW. Using the similar approach described here, we arrived at a new optimised Model 4 with 59\% bandwidth, if $L_{\mathrm{j}}$ is reduced to $64.4 \mathrm{pH}$ and $C_{\mathrm{s}}$ down to $26.3 \mathrm{fF}$. The required number of junction is now increased to close to 2,000 though.

\subsection{Performance Comparisons}

Fig. 4 (a) shows the gain curves calculated using the CME for all the models presented above, along with the curve from the original model. Model 1 requires only 500 instead of 2,000 JJs in order to achieve the same peak gain as the original model, which constitutes $75 \%$ reduction in the number of junctions required. The bandwidth is also improved by $29 \%$. Model 2 further increases the $\% \mathrm{BW}$ to $100 \%$, but requires $1,713 \mathrm{JJs}$, although much more than Model 1 but still slightly less than the original model. Nevertheless, both Model 1 and 2 suffer from having a low characteristic impedance, hence would only be useful for applications that favour low $Z_{0}$ such as transition edge sensor's readout. Model 3 and 4 were optimised for $Z_{0}=50 \Omega$, with the former opt for less junctions and the latter for larger bandwidth. For comparison, Model 3 requires only 594 junctions to achieve $9.3 \mathrm{~dB}$ peak gain but Model 4 requires closed to 2,000. However, the bandwidth is improved from $38 \%$ (similar the original model) to 59\%. The main characteristics of these models are summarised in Tab. 1. With this exercise, we have shown that depending on the targeted applications, there are flexibility for adjusting the design parameters of the JTWPA to suit to a specific requirement, be it opting for ease of fabrication with less junctions or broader operational bandwidth, or a compromise between the two.

\subsection{Higher harmonic suppression}

For an appropriate operation of a TWPA, the higher harmonics of the strong pump needs to be suppressed, and as we mentioned earlier, we achieve this by controlling the circuit cutoff frequency to be closed to the third harmonic $(\mathrm{TH})$ of the pump. However, as shown earlier, this may not always be the case for the various optimised models we presented earlier. As shown in Tab. 1, the cutoff frequency to pump frequency ratio $\left(f_{\mathrm{c}} / f_{\mathrm{p}}\right)$ 

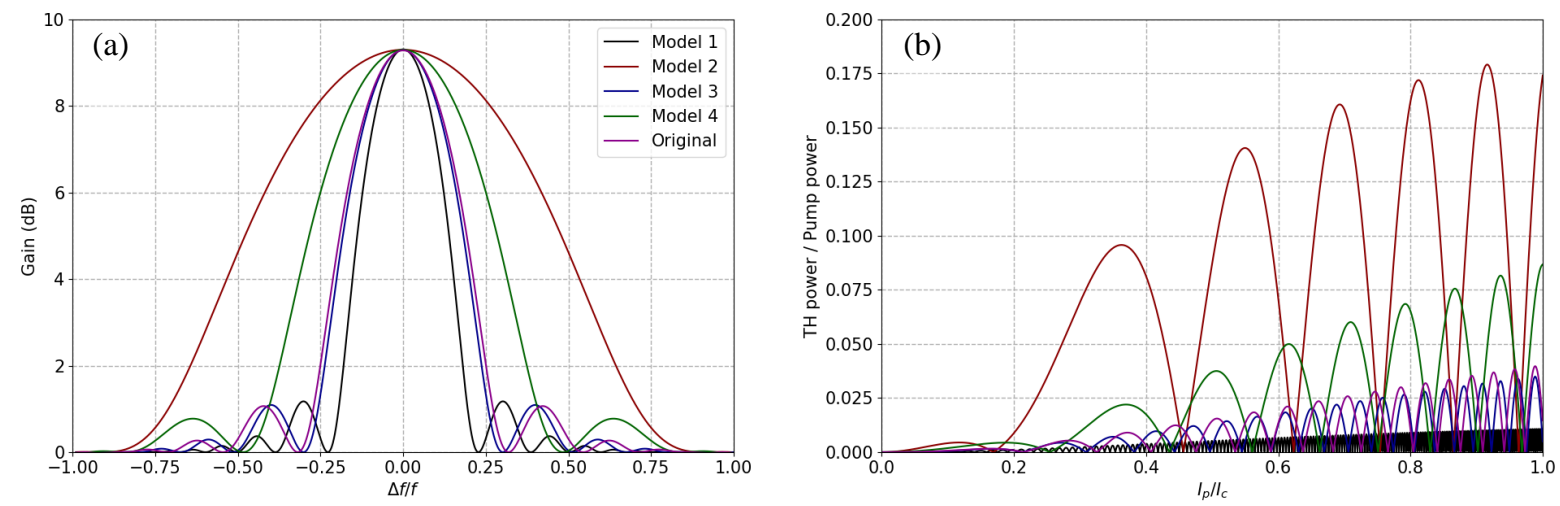

Figure 4. (a) Gain curves calculated using CME for our models and the original model. All the models use $I_{\mathrm{p}} / I_{c}=0.039$ and $I_{\mathrm{s}} / I_{\mathrm{p}}=0.5$. Model 1 parameters: $C_{\mathrm{j}}=240 \mathrm{fF}, C_{\mathrm{s}}=150 \mathrm{fF}$ and $L_{\mathrm{j}}=101.7 \mathrm{pH}$. Model 2 parameters: $C_{\mathrm{j}}=70 \mathrm{fF}$, $C_{\mathrm{s}}=290 \mathrm{fF}$ and $L_{\mathrm{j}}=9 \mathrm{pH}$. Model 3 parameters: $C_{\mathrm{j}}=70 \mathrm{fF}, C_{\mathrm{s}}=80 \mathrm{fF}$ and $L_{\mathrm{j}}=189.3$ pH. Model 4 parameters: $C_{\mathrm{j}}=70 \mathrm{fF}, C_{\mathrm{s}}=26.3 \mathrm{fF}$ and $L_{\mathrm{j}}=64.6 \mathrm{pH}$. the original model parameters: $C_{\mathrm{j}}=329 \mathrm{fF}, C_{\mathrm{s}}=39 \mathrm{fF}$ and $L_{\mathrm{j}}=100 \mathrm{pH}$. $(\mathrm{b})$ The generated third harmonic amplitude normalised to the pump current amplitude plotted against the strength of the pump current.

can be ranged from 3.02 (closed to the third harmonic) to 14.12. Hence, we further simulate the amplitude of the generated third harmonic for all our models. We focus only on the third harmonic because once the third harmonic is substantially attenuated, other higher harmonics and shock wave will be prevented from forming within the transmission line.

Fig. 4 (b) shows the amplitude of the third harmonic normalised to the pump amplitude plotted against pump current. Firstly, we note that the third harmonic amplitude is relatively weak for all the models, as long as the JTWPA is not pumped at higher current level. Compared to the original model, our Model 1 and 3 have a similar or lower level of third harmonic power, whilst Model 2 and 4 slightly higher. This is expected as Model 1 and 3 have a similar $f_{\mathrm{c}} / f_{\mathrm{p}}$ as the original model, while for Model 2 and 4 it is closed to $2-3 \times$ this ratio. Macklin et. al. ${ }^{9}$ experimentally proved that the higher harmonics of the pump can be effectively suppressed using the cutoff frequency for a JTWPA with $f_{\mathrm{c}} / f_{\mathrm{p}}=10.1$. As Model 1,3 and 4 have a lower $f_{\mathrm{c}} / f_{\mathrm{p}}$ ratio, we can be confident that the third harmonic will be suppressed in these model. However, Model 2 has a higher ratio of $f_{\mathrm{c}} / f_{\mathrm{p}}=14.12$, hence much higher third harmonic power. This undesired harmonic formation can be further repressed by placing a perturbing element along the main transmission line at every $\lambda / 2$ of the third harmonic frequency. In the case of resonator-coupled JTWPA which will be described later, these perturbation element can be the resonators themselves. The existence of these perturbing elements will create a small disturbance to the characteristic of the main transmission line, hence will create a periodically loading effect that produce a weak stopband at $3 f_{\mathrm{p}}$. This attenuation, combined with the circuit intrinsic attenuation due to the cutoff frequency will therefore be sufficient to suppress the formation of the third harmonic.

It is important to stress that with a pump to critical current ratio used throughout this paper $\left(I_{\mathrm{p}} / I_{\mathrm{c}}=0.039\right)$, the third harmonics generation is almost negligible. An interesting observation here is the location of the third harmonic peaks, which tents to shift towards higher pump power with higher $f_{\mathrm{c}} / f_{\mathrm{p}}$ ratio. This is advantages because it means that with higher $f_{\mathrm{c}} / f_{\mathrm{p}}$ ratio, the first peak will only arise at higher pump current, hence allowing a wider range of pump current to be used before exciting the third harmonic mode.

\section{OPTIMISED JTWPA WITH EXPONENTIAL GAIN}

Once we have optimised the circuit parameters to achieve various optimisation targets, it is easy to increase the peak gain by utilising dispersion control features to achieve exponential instead of quadratic gain. ${ }^{13}$ These dispersion engineered structure can be realised using periodically loaded elements, ${ }^{10,17-21}$ or high-Q resonators which was termed as Resonance Phase Matching (RPM) technique. ${ }^{9,13,14}$ We opt for the latter option for our 

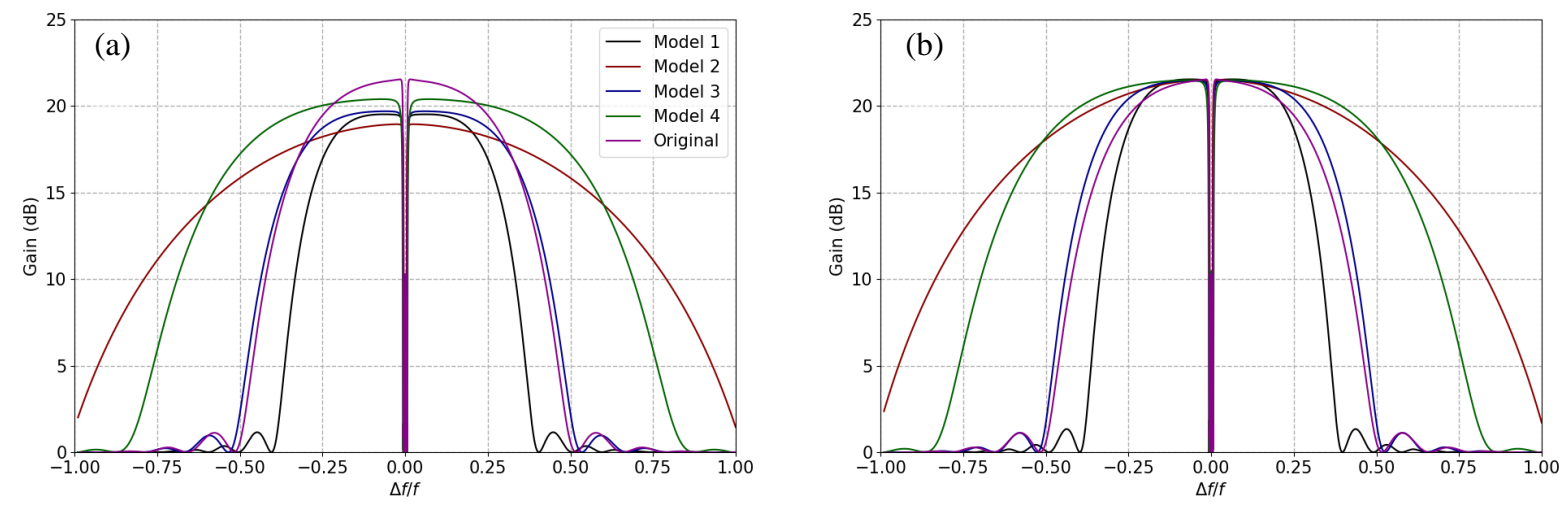

Figure 5. Theoretical gain curves of our models with RPM compared with the original theoretical design presented by O'Brien et. al. The resonator's parameters for Model 1: $C_{\mathrm{c}}=11.4 \mathrm{fF}, C_{\mathrm{r}}=2.53 \mathrm{pF}, L_{\mathrm{r}}=100 \mathrm{pH} ; \mathrm{Model} 2: C_{\mathrm{c}}=12.5 \mathrm{fF}$, $C_{\mathrm{r}}=2.53 \mathrm{pF}, L_{\mathrm{r}}=100 \mathrm{pH} ;$ Model 3: $C_{\mathrm{c}}=8.55 \mathrm{fF}, C_{\mathrm{r}}=2.53 \mathrm{pF}, L_{\mathrm{r}}=100 \mathrm{pH} ;$ and Model $4: C_{\mathrm{c}}=5.5 \mathrm{fF}, C_{\mathrm{r}}=2.53 \mathrm{pF}$, $L_{\mathrm{r}}=100 \mathrm{pH}$. The original model resonator's parameters are $C_{\mathrm{c}}=10 \mathrm{fF}, C_{\mathrm{r}}=7.036 \mathrm{pF}, L_{\mathrm{r}}=100 \mathrm{pH}$. (a) Gain curves with RPM with the number of junctions and the circuit parameters remain unchanged from the non-dispersion engineered models. (b) Updated gain profile with increased $N_{\mathrm{JJ}}$ to achieve the same peak gain as the original model.

JTWPA because the high junction inductance results in shorter transmission length (i.e., less number of unit cells required), and hence there will be insufficient number of repeated cells to accumulate the required dispersion divergence. Furthermore, resonators induce higher magnitude of dispersion deviation with a narrower stopband, thereby reducing the unusable bandwidth near the centre of the band.

We therefore added an $L C$ resonator to the original unit cell of our model, represented by a capacitance $C_{\mathrm{r}}$ connected in parallel with an inductance $L_{\mathrm{r}}$, which are shown as the red colour components in Fig. 1(b). The coupling between the main transmission line and the resonator is modelled by a coupling capacitance $C_{\mathrm{c}}$. The values of the resonator's parameters are chosen such that the resonance frequency $f_{\mathrm{r}}=10 \mathrm{GHz}$, and $C_{\mathrm{c}}$ is then optimised to maximise the gain peak as well as obtaining a flatter gain profile. Fig. 5 shows the gain curves calculated for all our models with the pump frequency fixed at $9.95 \mathrm{GHz}$ and a pump current of $0.039 I_{c}$.

Fig. 5 (a) shows the gain-bandwidth product calculated for our RPM models, utilising exactly the same optimised circuit parameters and the required number of junctions presented earlier. Here, one notes immediately that the peak gains of all our RPM models are slightly lower in comparison to the original model, as we also try to achieve flatter gain near the peak. It is well-known that the JTWPA gain can be controlled easily with the amplitude of the pump current, but in order to better compare the performance of all the RPM models against the original model, we chose to relax the originally value of $N_{\mathrm{JJ}}$ (i.e., the transmission line length) to reach the same peak gain of $21.5 \mathrm{~dB}$ as the original model. This results in a set of new $N_{\mathrm{JJ}}$ value i.e., 542, 1,890, 635 and 2,095 for Model 1, 2, 3 and 4 respectively. The gain profiles of these updated RPM models are shown in Fig. 5 (b). We can clearly see now that the bandwidth performance of all our RPM models are better than the the original model, except Model1. Furthermore, all the RPM models require less number of junctions than the the original model to achieve the same peak gain except Model 4 which have a similar $N_{\mathrm{JJ}}$ as the the original model. The main characteristic of these RPM models are also included in Tab. 1 for ease of comparison.

Although all these models could be useful for different applications, in our case it is important to have a $Z_{0}=50 \Omega$ amplifier, since it will be integrated as part of the dark matter search receiver. Therefore, it is fairer to compare only our $50 \Omega$ models with the original model. Here we clearly see that with Model 3 , we can achieve the same peak gain as the original model, with a slight improvement in bandwidth performance even thought it is marginal. However, the model requires only 542 tunnel junctions instead of 2,000 junctions, reducing $N_{\mathrm{JJ}}$ dramatically and hence producing a much compact and potentially less lossy JTWPA. On the other hand, if we opt for similar $N_{\mathrm{JJ}}$ as the original model, our Model 4 can achieve the same peak gain with much broader bandwidth, hence potentially can cover wider searching range for the dark matter search experiments. In short, 


\begin{tabular}{|l|c|c|c|c|c|}
\cline { 2 - 6 } \multicolumn{1}{c|}{} & Model 1 & Model 2 & Model 3 & Model 4 & Original \\
\hline$f_{\mathrm{p}}(\mathrm{GHz})$ & 9.95 & 9.95 & 9.95 & 9.95 & 5.97 \\
\hline$I_{c}(\mu \mathrm{A})$ & 3.24 & 36.61 & 1.74 & 5.1 & 3.29 \\
\hline$f_{c} / f_{\mathrm{p}}$ & 3.02 & 14.12 & 3.88 & 7.2 & 4.57 \\
\hline$a / \lambda(\%)$ & 4.1 & 1.6 & 4.0 & 1.3 & 1.21 \\
\hline$Z_{0}(\Omega)$ & 27.4 & 5.6 & 50.0 & 50.0 & 51.9 \\
\hline$P_{1 d B m}(\mathrm{dBm})$ & -102.6 & -88.5 & -105.4 & -96.1 & -99.7 \\
\hline$N_{\mathrm{JJ}}-$ without RPM & 502 & 1713 & 594 & 2010 & 2000 \\
\hline$N_{\mathrm{JJ}}-$ with RPM & 542 & 1890 & 635 & 2095 & 2000 \\
\hline Peak gain - without RPM $(\mathrm{dB})$ & 9.3 & 9.3 & 9.3 & 9.3 & 9.3 \\
\hline Peak gain - with RPM $(\mathrm{dB})$ & 21.5 & 21.5 & 21.5 & 21.5 & 21.5 \\
\hline \%BW - without RPM & 0.29 & 1.0 & 0.38 & 0.59 & 0.4 \\
\hline \%BW - with RPM & 1.18 & 1.63 & 0.87 & 1.37 & 0.83 \\
\hline
\end{tabular}

Table 1. Comparison of main characteristics of our JTWPA designs and the original model.

we successfully reduce the $N_{\mathrm{JJ}}$ needed for the original model substantially while maintaining a similar gainbandwidth performance, as well as designed an ultra-broadband JTWPA which have almost $1.5 \times$ bandwidth wider than the original model. These models can be further optimised to suit the axion dark matter search experiments, once the design of the search receiver is determined.

\section{CONCLUSION}

We have presented an in depth analyses of the impact of the different circuits parameters on the performance of a JTWPA. We have discussed the strategies to reduce the number of Josephson junctions required for a certain gain, how to increase the bandwidth and achieving a targeted characteristic impedance. Based on these analyses, we have designed different models optimised for different goals, and we compared the performance of our designs with the model presented by O'Brien et. al. We concluded our paper with two optimised models that have a $Z_{0}=50 \Omega$ characteristic impedance. In the first model, we successfully reproduce the gain-bandwidth product of O'Brien's model, but with significantly less number of tunnel junctions required. In the second case, using the same number of junctions as O'Brien et. al., we managed to broaden the operational bandwidth by closed to $65 \%$ while retaining the same peak gain. These optimised designs can be further altered to achieve a balance between the required number of junction and its operational bandwidth performance, once the dark matter search experiment specification is finalised.

\section{ACKNOWLEDGMENTS}

The authors would like to thank UKRI Quantum Technology for Fundamental Physics programme under the project Quantum Sensor for Hidden Sector, the European Research Council (ERC) programme under the project number 803862 (SPA4AstroQIT) and the Foundation MERAC for supporting this research work. J. M. Navarro's Ph.D. studentship is partly supported by the UK Science and Technology Facilities Council.

\section{REFERENCES}

[1] R. Khatiwada, D. Bowring, A. Chou, A. Sonnenschein, W. Wester, D. Mitchell, T. Braine, C. Bartram, R. Cervantes, N. Crisosto, et al., "Axion dark matter experiment: Detailed design and operations," arXiv preprint arXiv:2010.00169, 2020.

[2] T. Braine, R. Cervantes, N. Crisosto, N. Du, S. Kimes, L. Rosenberg, G. Rybka, J. Yang, D. Bowring, A. Chou, et al., "Extended search for the invisible axion with the axion dark matter experiment," Physical review letters 124(10), p. 101303, 2020.

[3] A. G. Droster and K. van Bibber, "Haystac status, results, and plans," arXiv preprint arXiv:1901.01668, 2019. 
[4] K. Backes, D. Palken, S. Al Kenany, B. Brubaker, S. Cahn, A. Droster, G. C. Hilton, S. Ghosh, H. Jackson, S. Lamoreaux, et al., "A quantum enhanced search for dark matter axions," Nature 590(7845), pp. 238-242, 2021.

[5] D. Schuster, A. A. Houck, J. Schreier, A. Wallraff, J. Gambetta, A. Blais, L. Frunzio, J. Majer, B. Johnson, M. Devoret, et al., "Resolving photon number states in a superconducting circuit," Nature 445(7127), pp. 515-518, 2007.

[6] M. Mirhosseini, A. Sipahigil, M. Kalaee, and O. Painter, "Superconducting qubit to optical photon transduction," Nature 588(7839), pp. 599-603, 2020.

[7] A. Opremcak, I. Pechenezhskiy, C. Howington, B. Christensen, M. Beck, E. Leonard, J. Suttle, C. Wilen, K. Nesterov, G. Ribeill, et al., "Measurement of a superconducting qubit with a microwave photon counter," Science 361(6408), pp. 1239-1242, 2018.

[8] A. V. Dixit, S. Chakram, K. He, A. Agrawal, R. K. Naik, D. I. Schuster, and A. Chou, "Searching for dark matter with a superconducting qubit," Physical review letters 126(14), p. 141302, 2021.

[9] C. Macklin, K. O'Brien, D. Hover, M. Schwartz, V. Bolkhovsky, X. Zhang, W. Oliver, and I. Siddiqi, "A near-quantum-limited josephson traveling-wave parametric amplifier," Science 350(6258), pp. 307-310, 2015.

[10] B. H. Eom, P. K. Day, H. G. LeDuc, and J. Zmuidzinas, "A wideband, low-noise superconducting amplifier with high dynamic range," Nature Physics 8(8), pp. 623-627, 2012.

[11] M. Kjaergaard, M. E. Schwartz, J. Braumüller, P. Krantz, J. I.-J. Wang, S. Gustavsson, and W. D. Oliver, "Superconducting qubits: Current state of play," Annual Review of Condensed Matter Physics 11, pp. 369$395,2020$.

[12] S. Chaudhuri, J. Gao, and K. Irwin, "Simulation and analysis of superconducting traveling-wave parametric amplifiers," IEEE Transactions on Applied Superconductivity 25(3), pp. 1-5, 2014.

[13] K. O'Brien, C. Macklin, I. Siddiqi, and X. Zhang, "Resonant phase matching of josephson junction traveling wave parametric amplifiers," Physical review letters 113(15), p. 157001, 2014.

[14] T. White, J. Mutus, I.-C. Hoi, R. Barends, B. Campbell, Y. Chen, Z. Chen, B. Chiaro, A. Dunsworth, E. Jeffrey, et al., "Traveling wave parametric amplifier with josephson junctions using minimal resonator phase matching," Applied Physics Letters 106(24), p. 242601, 2015.

[15] B.-K. Tan and G. Yassin, "Design of a uniplanar resonance phase-matched josephson traveling-wave parametric amplifier," in 2017 10th UK-Europe-China Workshop on Millimetre Waves and Terahertz Technologies (UCMMT), pp. 1-4, IEEE, 2017.

[16] J. Longden, F. Boussaha, C. Chaumont, K. Ratter, and B.-K. Tan, "Preliminary Characterisation of Titanium Nitride Thin Film at $300 \mathrm{mK}$ for the Development of Kinetic Inductance Travelling Wave Parametric Amplifiers," in Proc. SPIE Photonex + Vacuum Technologies 2021, (Scotland, UK), 2021. Submitted for publication.

[17] S. Chaudhuri, D. Li, K. Irwin, C. Bockstiegel, J. Hubmayr, J. Ullom, M. Vissers, and J. Gao, "Broadband parametric amplifiers based on nonlinear kinetic inductance artificial transmission lines," Applied Physics Letters 110(15), p. 152601, 2017.

[18] S. Shu, N. Klimovich, B. H. Eom, A. Beyer, R. B. Thakur, H. Leduc, and P. Day, "Nonlinearity and wideband parametric amplification in a (nb, ti) n microstrip transmission line," Physical Review Research 3(2), p. 023184, 2021.

[19] L. Planat, A. Ranadive, R. Dassonneville, J. P. Martínez, S. Léger, C. Naud, O. Buisson, W. HaschGuichard, D. M. Basko, and N. Roch, "Photonic-crystal josephson traveling-wave parametric amplifier," Physical Review X 10(2), p. 021021, 2020.

[20] M. Malnou, M. Vissers, J. Wheeler, J. Aumentado, J. Hubmayr, J. Ullom, and J. Gao, "Three-wave mixing kinetic inductance traveling-wave amplifier with near-quantum-limited noise performance," $P R X$ Quantum 2(1), p. 010302, 2021.

[21] K. Ratter and B.-K. Tan, "A Dispersion-Engineered Josephson Travelling Wave Parametric Amplifier with Periodic Impedance Perturbation," in Proc. $31^{\text {st }}$ Int. Sym. Space THz Technol. (ISSTT), (Tempe, Arizona, U.S.A.), 2020. . 